European Journal of

Technology

(EJT)

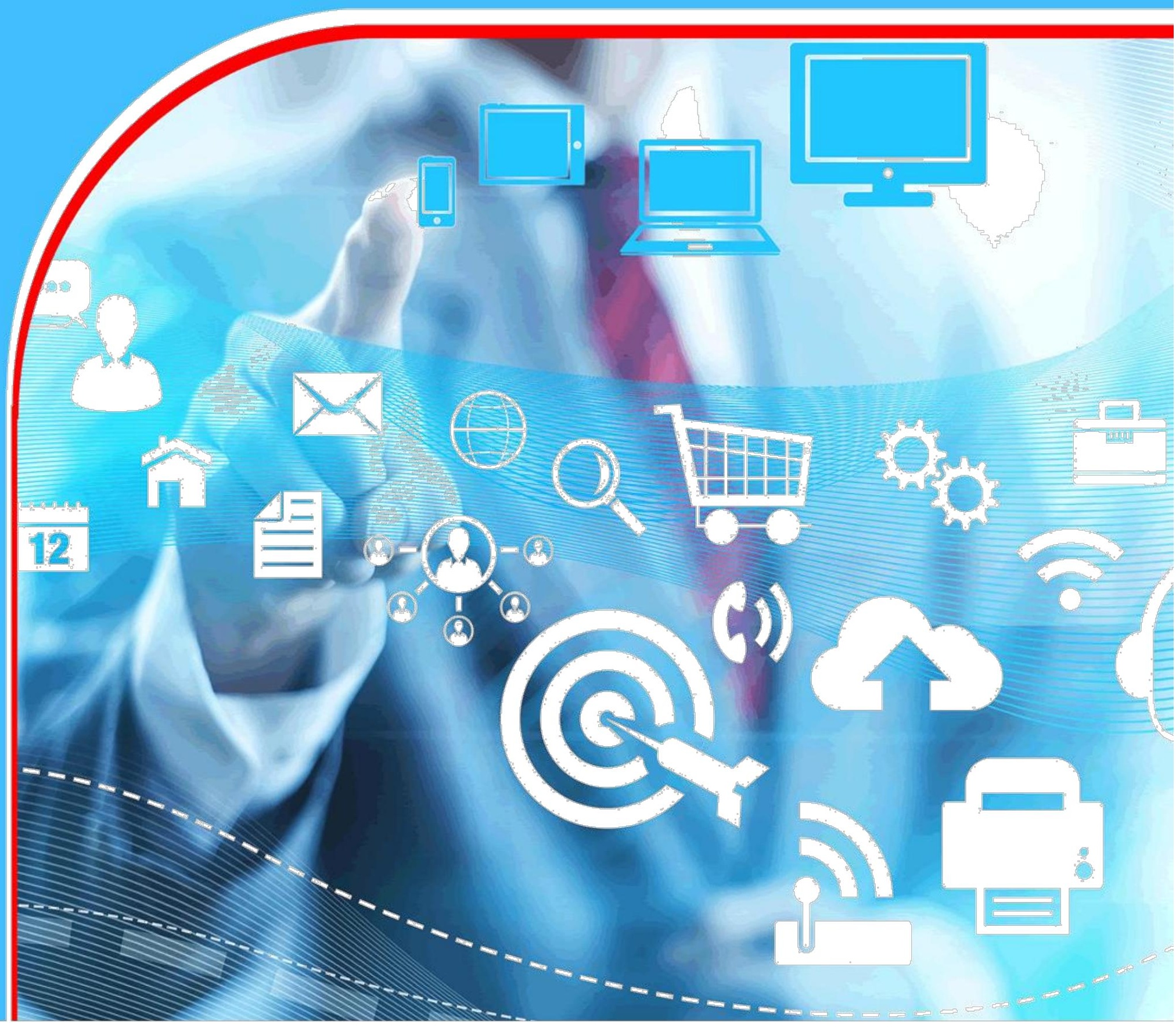

TRAFFIC DESIGN MODEL FOR OPTIMUM

PERFORMANCE ENHANCEMENTS OF IEEE 802.11B

QOS PARAMETERS

Chukwuedozie N. Ezema ${ }^{a}$, Chinazam C. Ezema, Chukwuebuka B. Umezinwa ${ }^{c}$ 


\title{
TRAFFIC DESIGN MODEL FOR OPTIMUM PERFORMANCE ENHANCEMENTS OF IEEE 802.11B QOS PARAMETERS
}

\author{
Chukwuedozie N. Ezema ${ }^{* a}$, Chinazam C. Ezema ${ }^{b}$, Chukwuebuka B. Umezinwa ${ }^{c}$ \\ a, b Department of Electronic and Computer Engineering, Nnamdi Azikiwe University Awka, \\ Anambra State, Nigeria. \\ ${ }^{\mathrm{c}}$ Department of Electronic and Computer Engineering, Imo State Polytechnic Umuagwo, \\ Imo State, Nigeria.
}

\begin{abstract}
Purpose: The objective of this research is to design a traffic model for optimum performance analysis and enhancements of IEEE $802.11 \mathrm{~b}$ QOS parameters. It investigated the effect of traffic distribution on the quality of service parameter on WLAN, in other to establish enhanced WLAN Performance. In order to evaluate the mean packet delay and network throughput performance, this research has presented a traffic loading for MAC DCF which supports WLAN's QOS Parameters.
\end{abstract}

Methodology: Based on this model, a computer simulation model over a MATLAB Simulink was developed.

Results: In this research, results show that the IEEE $802.11 \mathrm{~b}$ does not perform well in terms of high throughput, and low mean delay at high traffic load conditions. Furthermore, it was also shown that the mean packet delay of arrived packets decreases as the number of workstations decreases, but at saturation, it was shown that throughput decreases, mean packet delay increases. Therefore, to achieve a better enhanced network performance, it was observed that IEEE 802.11b WLAN QOS parameters can be improved to a maximum throughput.

Keywords: Distribution System, WLAN Architecture, Network Model, Network Throughput Performance, Quality of Service (QOS). 


\subsection{INTRODUCTION}

The design of robust and reliable networks and network services is becoming increasingly difficult in today's world. The only path to achieve this goal is to develop a detailed understanding of the traffic characteristics of the network. Managing performance of networks involves optimizing the way networks function in an effort to maximize capacity, minimize latency and offer high reliability regardless of bandwidth available and occurrence of failures [4], [9]. Network performance management consists of tasks like measuring, modeling, planning and optimizing networks to ensure that they carry traffic with the speed, capacity and reliability that is expected by the applications using the network or required in a particular scenario.

Networks are of different types and can be categorized based on several factors. However, the factors that affect the performance of the different networks are more or less the same. These involve parameters like Latency, Packet Loss and Throughput. In order to design high performance networks or guarantee performance of any type of network detailed analysis of the above factors is a crucial step. Often the foremost step in such an analysis is the study of the traffic on the network. As a consequence the type of traffic model used to understand the flow of traffic in the network, and how closely the model depicts the real-time characteristics of the network, become vital parameters. Choosing a model that does not describe the realtime characteristics of the traffic in the network can be as disastrous as not analysing the traffic at all.

The IEEE 802.11 architecture comprises several components and services that interact to provide wireless communication to stations which are any devices that incorporates the functionality of the IEEE 802.11 protocol and can connect to the wireless media. Supported services are authentication, de-authentication, privacy, and delivery of the data.

\subsection{Basic Service Set (BSS)}

The IEEE 802.11 standard specifies three primary setups. The first setup is a Basic Service Set (BSS), which is defined as a group of stations that communicates with each other in a geographical area known as Basic Service Area (BSA). When these stations can communicate without the aid of an infrastructure network, they are referred to as an Independent Basic Service Set (IBSS) which is the formal name of an ad hoc network in the IEEE 802.11 standard. These stations operate in the "ad hoc mode" because they communicate directly with another station in its transmission range. In comparison, in the "infrastructure mode", a station in a BSS communicates with another through a Base Station (BS) which is also called Access Point (AP) if it is connected to a wired network. The BSS operating with a BS is known as the Infrastructure Basic Service.

\section{2 Extended Service Set (ESS)}

An ESS is a set of infrastructure BSSs, where the APs communicate among themselves to forward traffic from one BSS to another and to facilitate the movement of mobile stations from one BSS to another. The APs perform this communication via an abstract medium (called the distribution system (DS)). To network equipment outside of the ESS, the ESS and all of its mobile stations appears to be a single MAC-layer network where all stations are physically stationary. Thus, the ESS hides the mobility of the mobile stations from everything outside the ESS. In this setup, BSs (or APs) provide the integration points for network connectivity among different BSSs. Therefore, a network backbone, also known as distribution system (DS), is formed. 


\subsection{Distribution System (DS)}

The distribution system (DS) is the mechanism by which one AP communicates with another to exchange frames for stations in their BSSs, forward frames to follow mobile stations from one BSS to another, and exchange frames with wired network. In the infrastructure mode, a station needs to join a BSS to communicate. It obtains synchronization information from periodic beacons from the Base Station. It can either obtain this information by requesting it from the BS (active probing), or it can wait for the periodic beacon from the Base Station. Before being able to send and receive data, the station has to go through an authentication and association process.

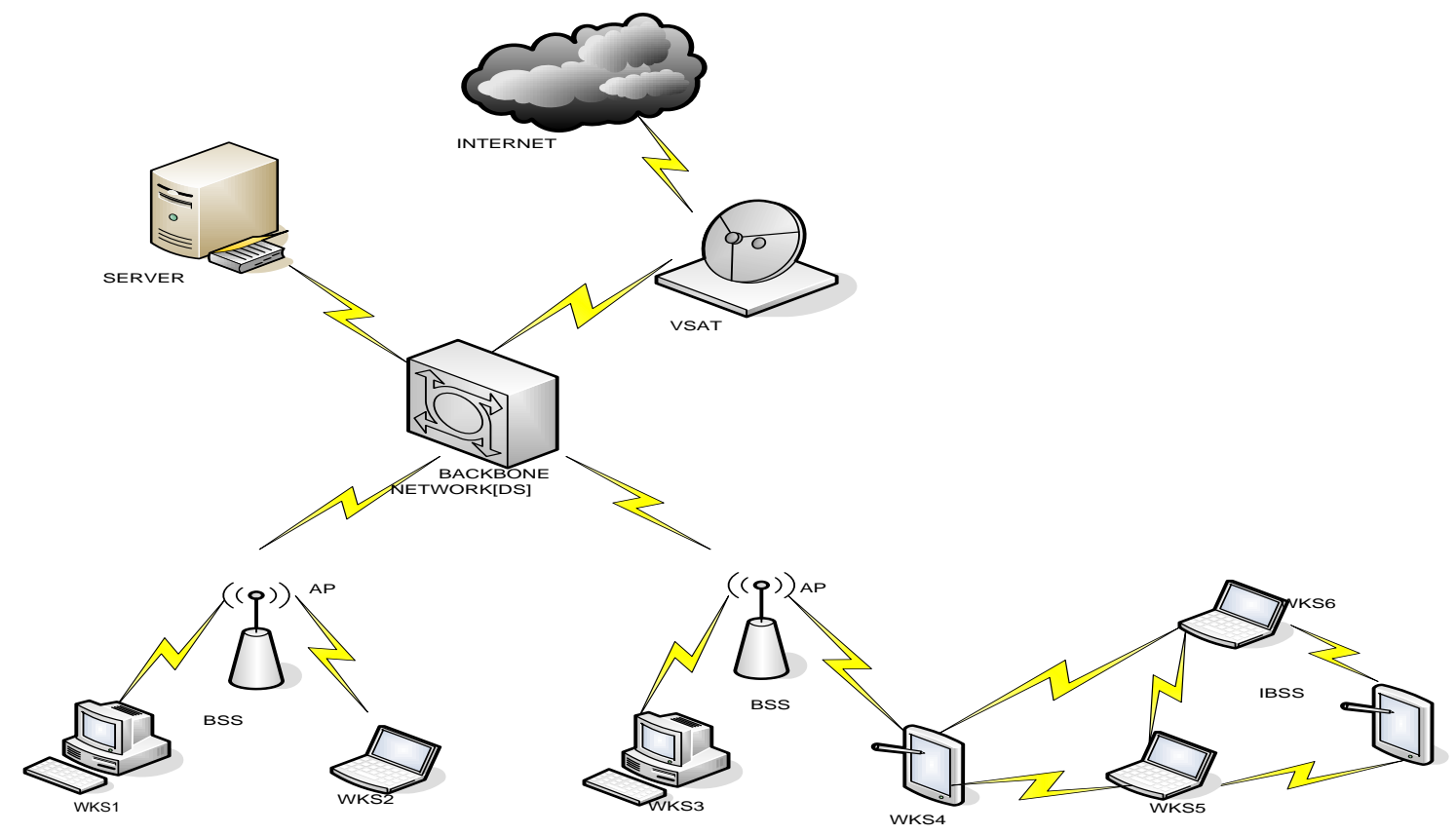

Fig 1: IEEE802.11 [WLAN] Architecture

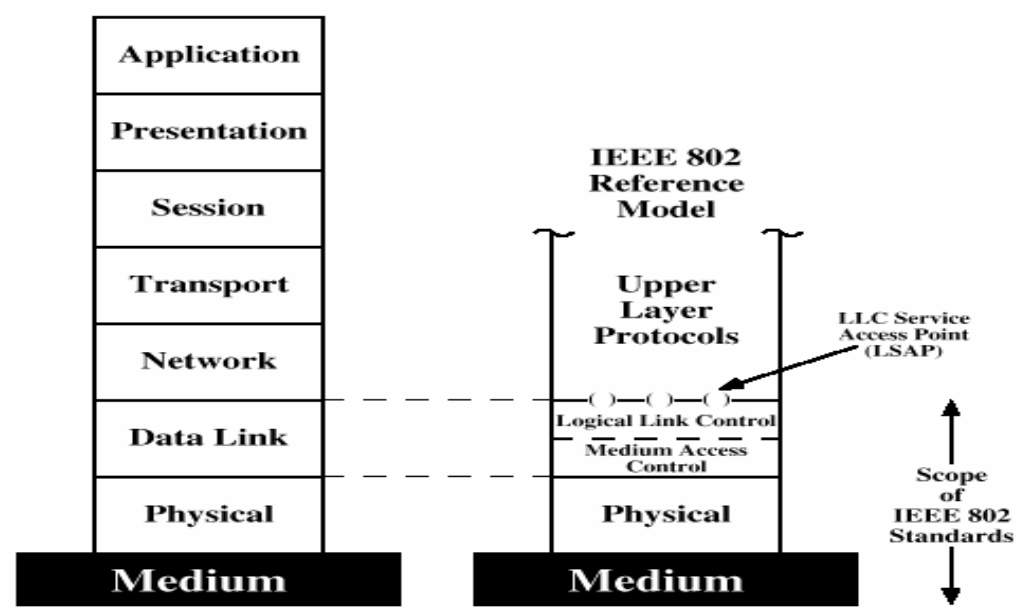

Fig 2: IEEE 802.11 Protocol Layers compared to OSI Model 


\subsection{The Adopted Typical WLAN Architecture}

This research focuses on the principles of Basic Access mechanism (CSMA/CA). In this section, the basics of the IEEE 802.11 standard are depicted. Many concepts and definitions are needed in order to better understand the IEEE 802.11 standard. The specifications details can be found in [19]. The IEEE 802.11 architecture comprises several components such as workstations, the WLAN Access Point (WLAN AP), Distribution System (DS), Server and Very Small Aperture Terminal (VSAT) and services that interact to provide wireless communication to stations which are any devices that incorporates the functionality of the IEEE $802.11 \mathrm{~b}$ protocol and can connect to the wireless media.

In the infrastructure mode, a station needs to join a BSS to communicate. It obtains synchronization information from periodic beacons from the BS. It can either obtain this information by requesting it from the BS (active probing), or it can wait for the periodic beacon from the BS. Before being able to send and receive data, the station has to go through an authentication and association process. [7] The BSS links the server or the VSAT through the distribution system (DS) while the ESS links the internet via the VSAT. The adopted WLAN architecture is depicted in the figure below.

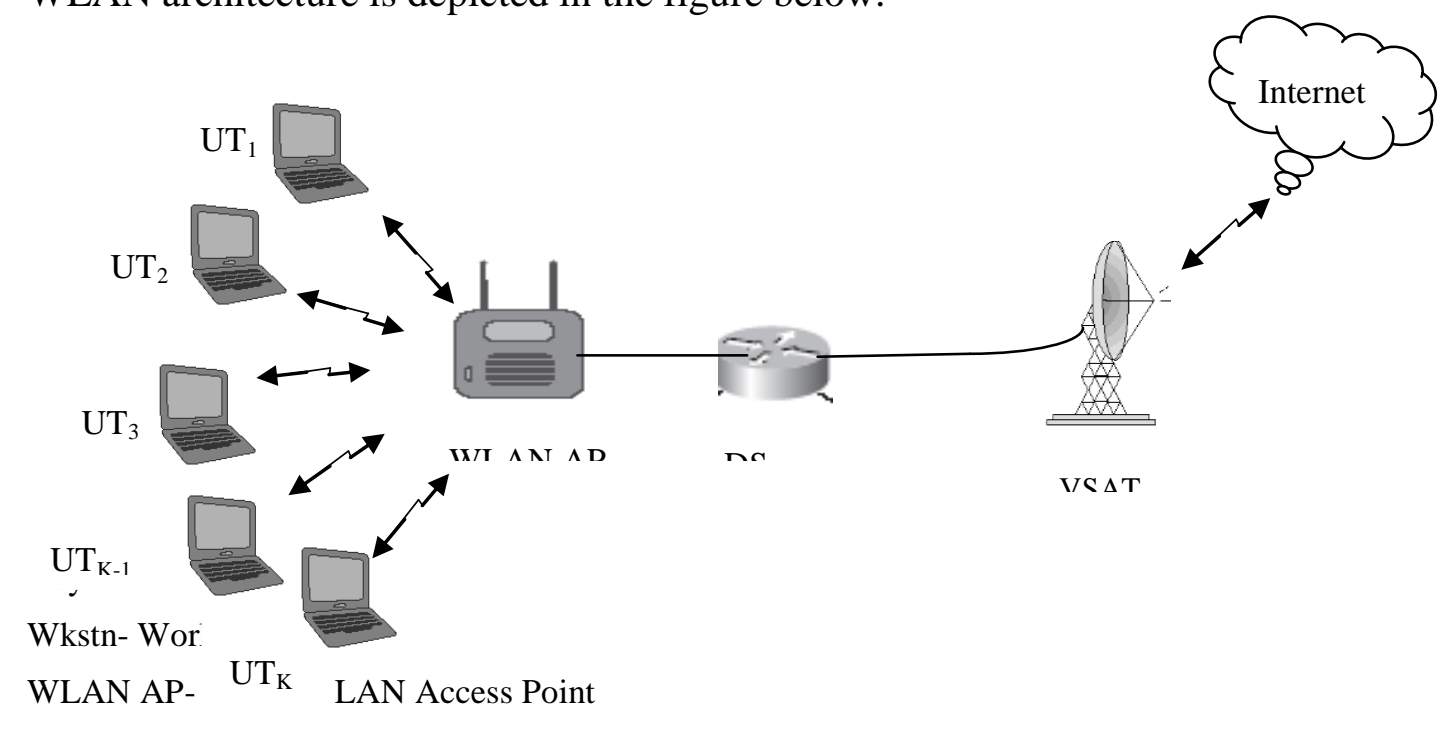

VSAT- Very small Aperture Terminal

\section{Figure 3: Adopted Typical WLAN Architecture}

\subsection{Operation of Basic Access Mechanism (CSMA/CA)}

The MAC protocol for the architecture was based on DCF which is usually implemented on Ad hoc network [10]. In DCF, The DCF protocol allows stations to access the medium in a distributed manner. There is no central entity controlling the use of the shared channel. The DCF is the basic access method used to support asynchronous data transfer on a best-effort basis. The DCF is based on the carrier sensing multiple accesses with collision avoidance (CSMA-CA) protocol. The CSMA/CA Media Access Control (MAC) protocol of IEEE WLAN is a unique way of avoiding the collision of the user packets when the traffic sources transmit bursty packets [11]. Carrier sensing involves monitoring the channel to determine whether the medium is idle or busy. If the medium is busy, it makes no sense for a station to transmit its frame and cause a collision and waste band-width. Instead the station should wait until the channel becomes idle. If it is still idle, the station sends its frame. Otherwise, the MAC selects a back-off value randomly from a contention window. Figure 4 shows this scheme. If a collision happens the contention window is set to twice its size and a back-off 
value is chosen from the new interval. After a successful transmission, the contention window is reset to a pre-set minimum value. The random back-off is also called after each successful transmission and each retransmission to reduce the probability of collisions.

The IEEE 802.11 MAC uses a positive acknowledgement scheme to detect collisions. Each unicast frame sent by the MAC has to be acknowledged by the receiver; otherwise the frame is retransmitted by the MAC layer. Broadcast packets are not acknowledged. Also, retransmissions are limited to a maximum number of tries, after which a packet is dropped. All stations are required to support the DCF because the access control in ad hoc networks uses only the DCF. Infrastructure networks can operate using just the DCF or a coexistence of the DCF and PCF [20].

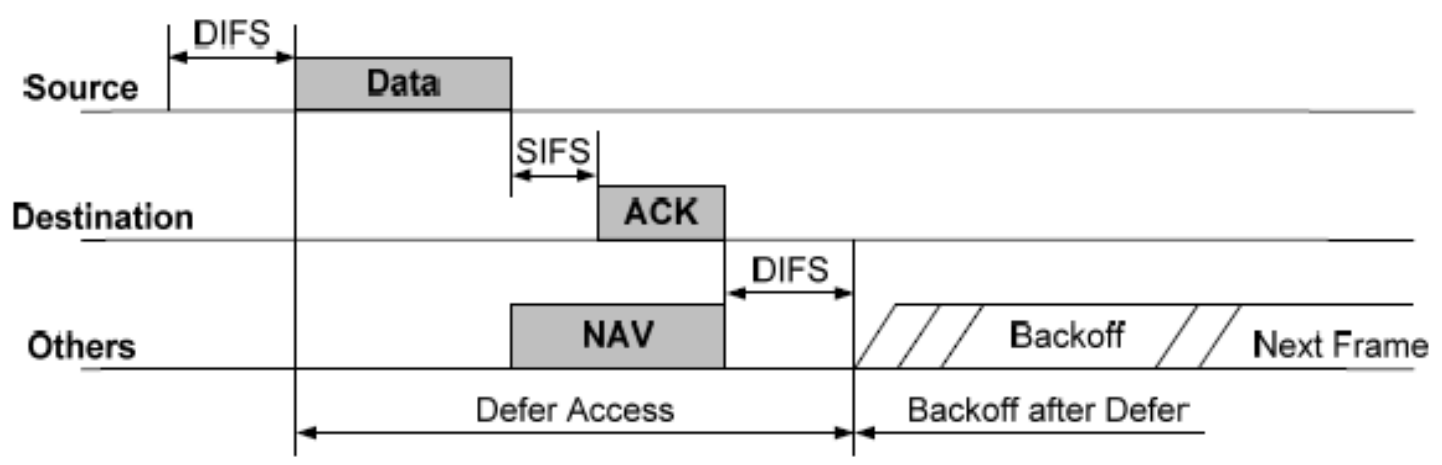

Fig.4: Basic access mechanism in DCF

\subsection{Network Model}

The performance of a WLAN may be defined by the QoS parameters. The QoS parameters considered in the modeling of the WLAN are packet delay, packet loss rate and throughput etc. These QoS parameters influence the operation of the network and specifically the resource assignment to the traffic load.

The network model was based on an isolated IEEE 802.11 MAC DCF protocol of the network architecture which operates on the principles of CSMA/CA. The figure 5 represents the network model

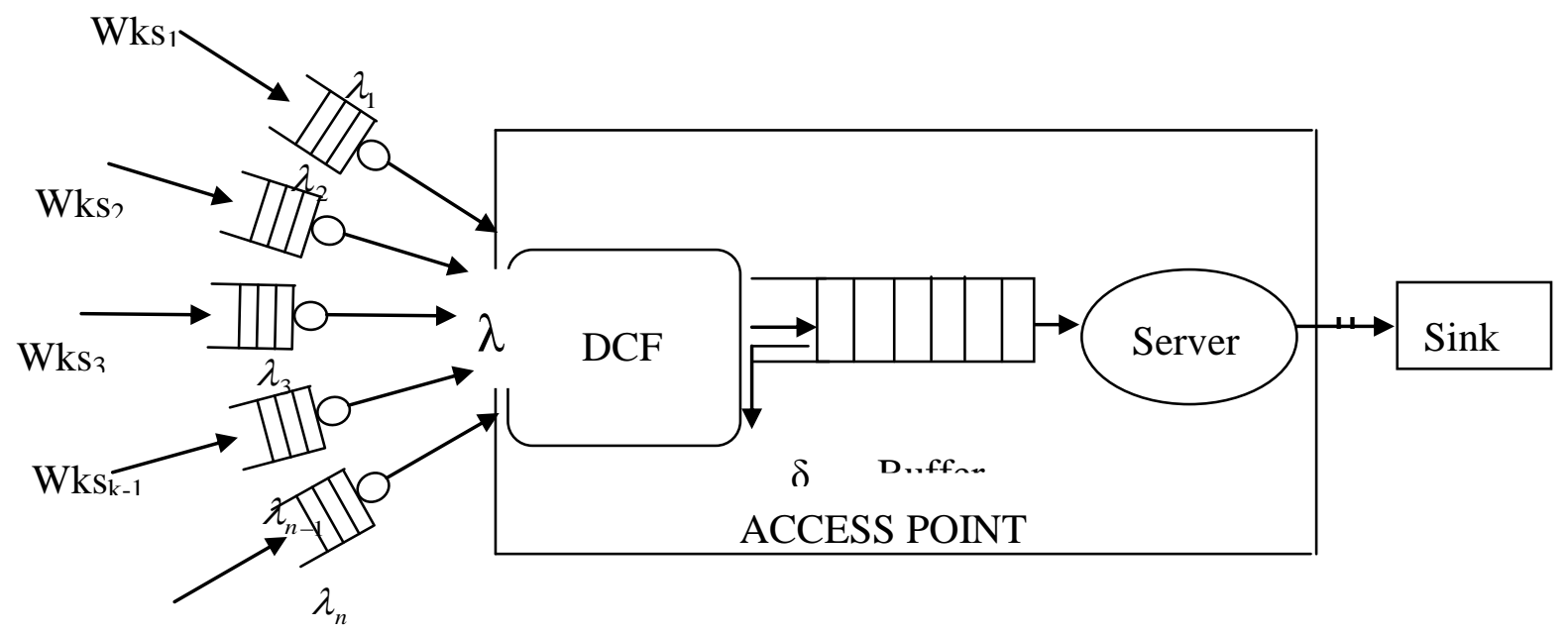

$\mathrm{Wks}_{\mathrm{k}}$

Where,

$\lambda=$ arrival rate,

$\mu=$ service rate 


\section{Figure 5: Model of WLAN Access Point}

Also, the entire communication network has severally been shown to be modeled as a single network node [15]. The WLAN model was defined to simply consist of workstations (traffic source generators and sinks), MAC DCF communication (CSMA/CA) protocol, single server and a buffer system. The source generators were intended to generate bursty packets with varied length randomly. The source generators were developed on the basis that packet arrival process is randomly distributed and Poisson in nature with intensity. The source generators have parameters such as arrival rate, retransmission, binary exponential back off counter and packet length. Also, random exponentially distributed packets lengths were implemented at the WLAN access point. The rate of packet transmission through the wireless medium was represented by $\mu$ (bps).

The MAC DCF communication protocol was employed in the simulation modeling in order to ensure that the arriving traffic has equal probability of being served in a random manner.

The WLAN server was modeled based on the principle of CSMA. The WLAN server serves packets for a period through the output port on a sequence of first-in-first-out (FIFO). The pattern of services follows exponential distribution. The server utilization was enhanced by the application of buffering system that controls packet arrivals from the sources. Collisions occur when two sources send data at the same time. The workstations use random back-off procedure to avoid probability of two or more workstations accessing the channel at the same time. The traffic intensity and the traffic trends of the WLAN were probed during and after simulation time.

The sink terminates packets transmitted from the source generators via the single server. The sink has stop time attached to it. The stop time gives the delay or time elapsed of the packets transmitted.

Probes were strategically implemented in the model to gather the necessary information (data) on the desired quality of service (QoS) parameters. In other words, the WLAN QoS parameters such as packet loss rate and packet delay are also probed and they can be displayed graphically or numerically.

\subsection{METHODOLOGY}

The approach used in the actualization of this research, is based on the principles of CSMA/CA using the MATLAB Simulink Environment. Subsequently, the physical architecture used for the modeling was converted into computer simulation model using MATLAB Simulink Environment. The computer simulation model is simulated, for Quality of service parameter and traffic distribution parameter collation.

Computer simulation is a very useful technique that can be used to model different kinds of systems including network computers leveraging inherent characteristics of computers such as its computational power. In this manner, one can simulate and experiment with multiple 
real or future scenarios in order to compare and develop solutions to improve systems performance [10]. Computer Simulation is a powerful and important tool because it provides a way in which alternate designs, models and methods can be evaluated without having to experiment on a real system, which could be costly or simply impossible because elements may not exist right now, but can be built in the future if feasible. Network designers and developers want assurances that their designs will achieve the desired goal prior to actual production and deployment. Network operators want assurances that the introduction of a new device type into their networks will have no unintended consequences. The available methods that provide these types of assurances include analysis, prototype development, empirical testing, low-scale trial deployments, modeling and simulation (M\&S) [10], [16]. Modeling and simulation (M\&S) are critical elements in the design, development, test and evaluation (T\&E) of any network product or solution. While not sufficient unto themselves, $M \& S$ are particularly valuable methods due to the fact that they $(M \& S)$ are, in most cases, the only viable methods to gain insight into the performance of the eventual product or solution in a large-scale environment prior to actual deployment. They are used for design trade studies and deployment decisions.

A simulation represents the execution of a model. It consists of a typical set of inputs, algorithms and routines that model the system behavior and a set of outputs that provide insight into the system performance. The systems can be as simple or as complex as the user desires [20].

Modeling and simulation (M\&S) methods are employed by scientists and engineers to gain insight into systems' behavior that can lead to faster product time-to-market and more robust designs [10]. These advantages come at the cost of model development time and the potential results are questionable as a result of the fact that the model represents limited attributes of the actual system. $M \& S$ techniques are used to characterize complex interactions and performance at various layers of the protocol stack.

The key parameters considered for the modeling of WLAN access point for enhanced performance at network saturation, are the packet arrival rate, mean packet delay and network throughput. A station that is ready to transmit packets generates a random number, calculates its exponential value of the back-off counter and keeps decreasing the value of its back-off counter after the air medium was sensed to be idle. Other parameters considered are the contention window, the number of workstation stations, the service rate and the number of attempts.

In this research, MATLAB was used to implement IEEE 802.11b MAC DCF Saturated traffic distribution. The model was converted into a computer simulation model using MATLAB Simulink package. Insight into the system's performance was provided by the probes strategically positioned to collect data for the calculation of the mean packet delay as expressed in equation (1) below. Byung-jack wak etal defined medium access delay as the time taken from the moment a packet is ready to be transmitted to the moment the packet starts its successful transmission [18]. Transmission of bits from workstations (sources) to the destination (sink) via the medium access-CSMA/CA controller was observed for $\mathrm{n}$ intervals and the average delay was taken. Network packet delay was considered as being comprised of packet access delay, propagation delay and processing delays. The average delay was processed as expressed in equation (1) below [8], [17]. 
Average delay $=\frac{\sum_{i=1}^{M} \text { delay }_{\mathrm{i}}}{M}-\mathrm{C}_{\mathrm{N}}$

Where,

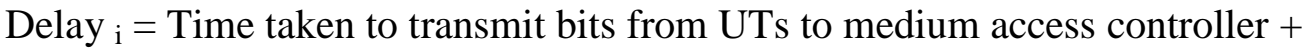

Time taken to send the bits from medium access controller to the destination + Time taken to retransmit bits due to collision

$=$ Time taken to offer bits - time taken to serve the bits

$\mathrm{M}=$ Total number of packets transmitted.

Throughput is defined as the number of bits passing through a point in a second or it is defined as the number of packet passing through the network in a unit time. [8]

Throughput $=$ Total number of bits offered by UTs - number of bits pending (unserved) number of bits dropped.

$$
\text { = }(\text { bits_offered }) \text { - (bits_pending }) \text { - (bits_dropped })
$$

\subsection{RESULTS AND DISCUSSIONS}

\subsection{Effect of Workstations on Network Throughput Performance}

Throughput: In radio communication networks, throughput or network throughput is defined as the average rate of successful message delivery over a communication channel. Data may be delivered through physical or logical link, or pass through a certain network node. Throughput is usually measured in bits per second (bit/sec). System throughput or Aggregate throughput is defined as sum of data rates that are delivered to all STA's within communication range. Throughput directly depends on control message overhead and congestion. As the control message overhead and collision increases overall throughput will be decreased.

Network Load: Bandwidth offered by an Access Point is shared among all STA ${ }^{\text {ees }}$ within its coverage area. Throughput of an Access Point increases in proportion to the amount of packet traffic load added by all connected STA's provided that the overall traffic load does not exceed the capacity of the AP. When AP's workload exceeds or approaches its capacity, the throughput does not increase further. This phenomenon is called channel Saturation or congestion. In general, highly overloaded AP may simply reject new association requests. If an AP is not overloaded then it grants association request from STA's based on work-load status [9]. The request can be granted only when the predicted load level after the association does not exceed some predefined threshold. A simple way to increase overall system throughput is to deploy additional APs covering the same region, in anticipation that heavy traffic load can be distributed among multiple APs. Unfortunately, as each STA independently selects an AP to camp on, STA's may be associated with few APs while other APs remain idle. Consequently, the traffic load is not fairly shared by APs. This problem motivates load-balancing protocols for IEEE 802.11 networks.

In Fig. 6, 7 and 8, the graph of network throughput versus number of workstations for infrastructure networks were plotted. Considering the Gamma traffic distribution, which has the best performance and slightly different behaviour from the three distributions, it showed that throughput is higher and increases with continous increase in data rate but decreases 
slightly with increase in the number of workstations from $10-80$ workstations at $1,2,5.5,11$ Mbps data rate. In order words, it was observed that the network throughput decreases as the number of active stations increases for $\mathrm{N}=10$ to 80 stations at $80 \%$ offered load. It was also observed that the network throughput under the infrastructure network is reasonable, especially for $\mathrm{N}>30$ stations. Under the infrastructure network, the throughput is saturated at around $\mathrm{N} \geq 80$ stations. Now let us examine the maximum and minimum throughput of the IEEE $802.11 \mathrm{~b}$. The maximum achievable throughput is $4.6 \mathrm{Mbps}$ for $\mathrm{N}=10$ station at $80 \%$ offered load. This throughput is approximately $44 \%$ of the maximum theoretical bandwidth of $11 \mathrm{Mbps}$. The minimum throughput under the infrastructure network is $1.8 \mathrm{Mbps}$ which is around $16 \%$ of the maximum bandwidth of $11 \mathrm{Mbps}$ for $\mathrm{N}=80$ stations at $80 \%$ offered load. The throughput performance strongly depends on the number of the stations for all data rates when basic access mechanism is employed.

On the other hand, if basic access is used, the throughput decreases as the number of the stations increases because more collisions take place at that point. Moreover, the throughput is reduced when the data rate increases. The situation is explained by considering that the time spent for frame transmission is decreased as the data rate increases but the time overhead spent on DIFS, SIFS and the back off delay remains the same.

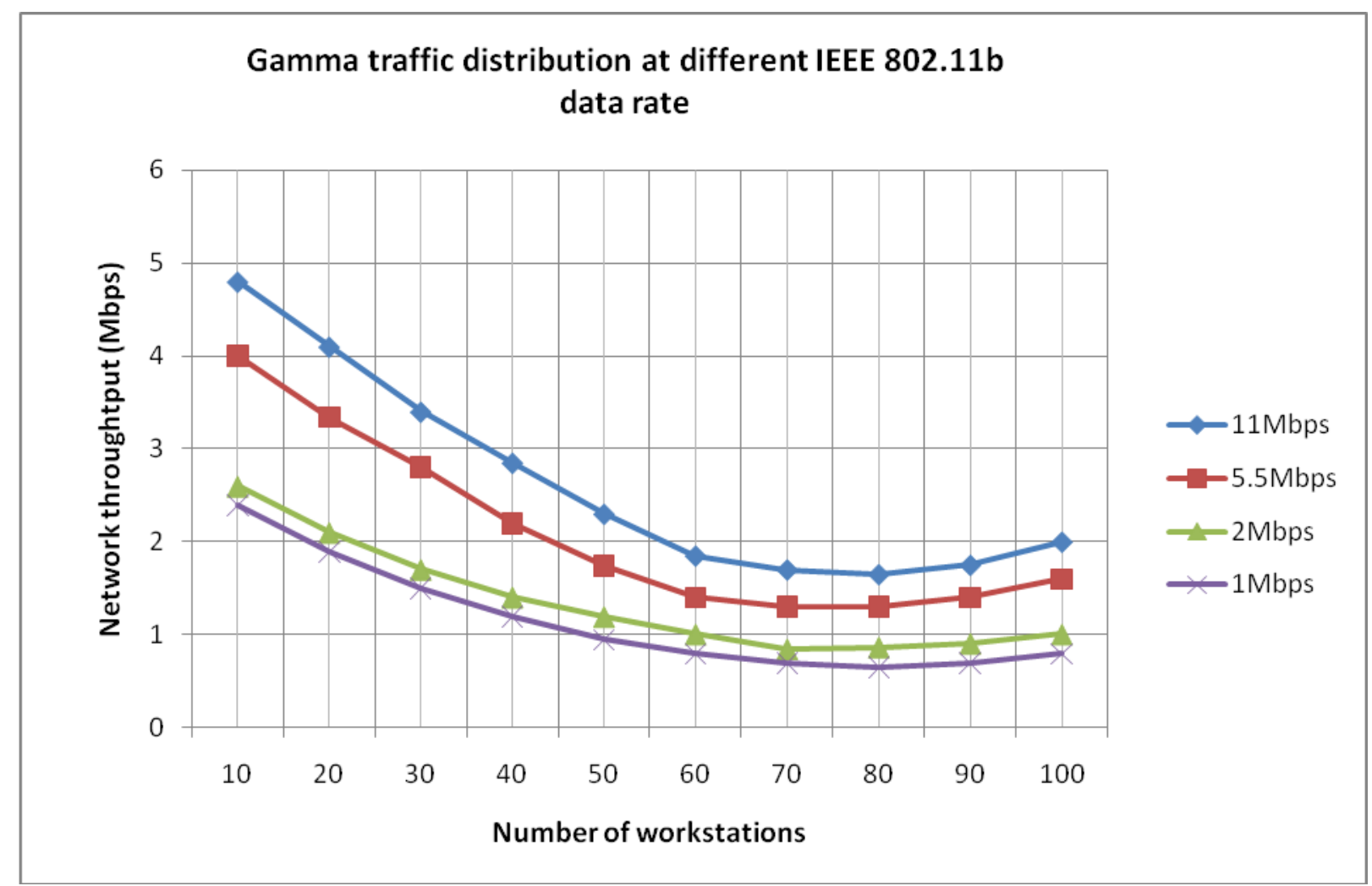

Figure 6: Graph of Mean Throughput against Number of workstations using Gamma traffic distribution 


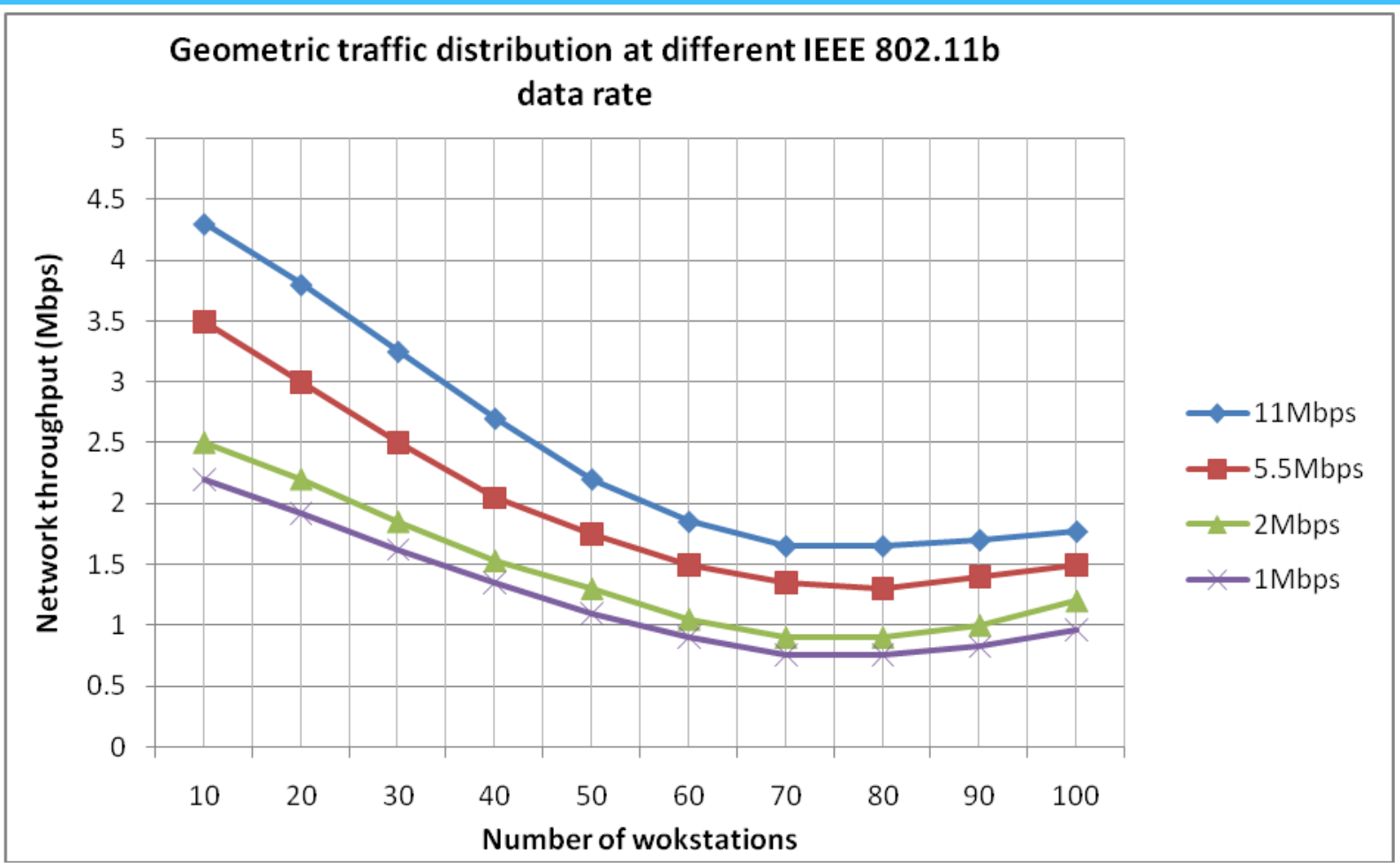

Figure 7: Graph of Mean Throughput against Number of workstations using Geometric traffic distribution

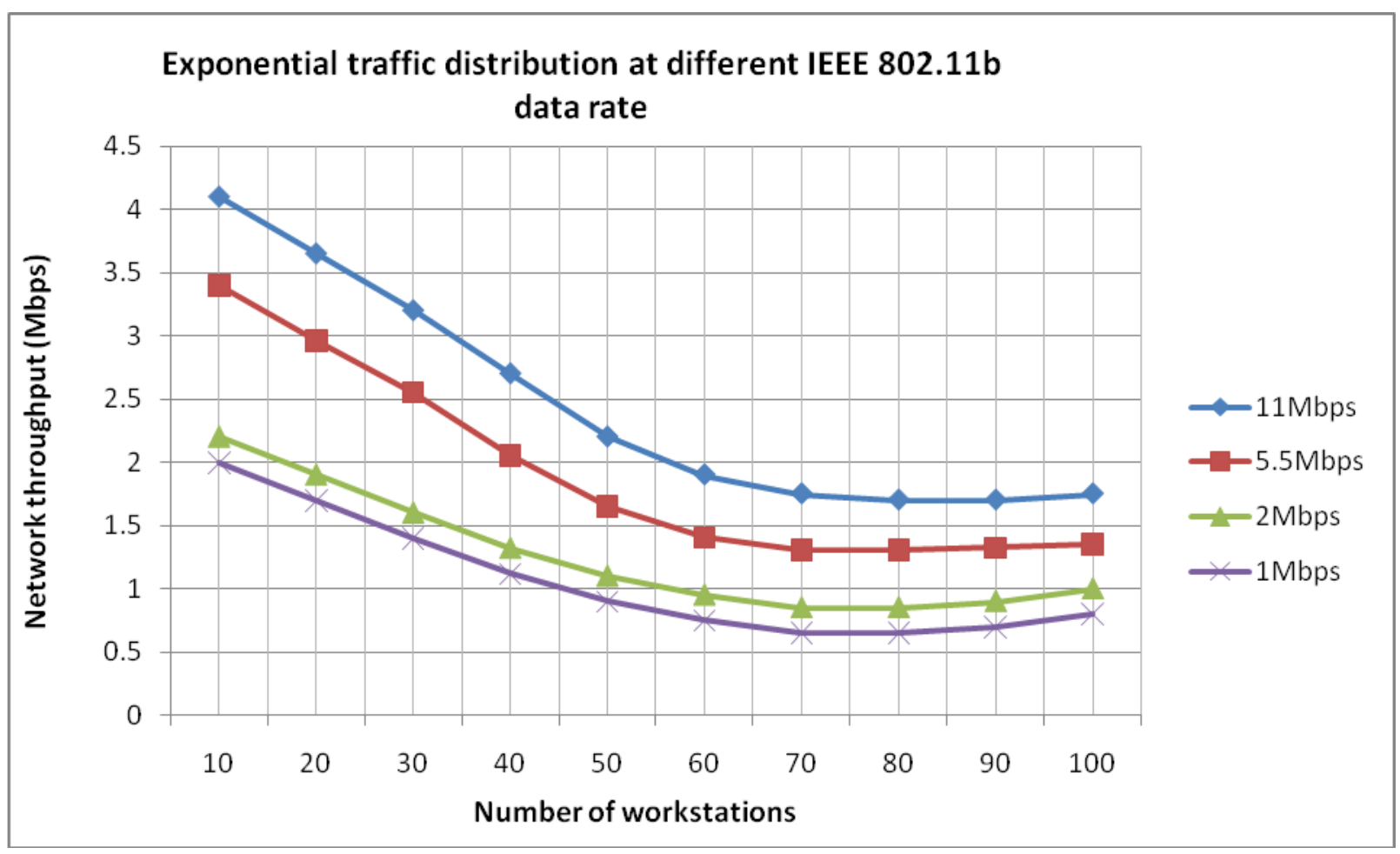

Figure 8: Graph of Mean Throughput against Number of Sources using Exponential traffic distribution 


\subsection{Effect of Workstations on Network Mean Delay Performance}

Mean Delay: Whenever data is being transmitted from sender to receiver there is delay due to resource scarcity. Usually there are different types of delays in communication network namely processing delay, queuing delay, transmission delay and propagation delay. when there are only 10 wireless LAN STAs then STA that want to access the media, does not need to wait longer, but when the wireless LAN stations increases, then the station that want to access media has to wait for longer time. When the STA's are 70 then the Media Access delay for one STA that want access media is much longer compared with other scenarios.

Network Load: Figure 9, 10 and 11 explain about network load for various scenarios. It shows that whenever, the number of wireless LAN station is increased, and then the load on Access Point will be increased. In Fig. 9, network mean packet delay versus number of workstations for infrastructure networks was depicted. It was observed that under infrastructure networks, the mean packet delay increases as I increase the number of workstations for $\mathrm{N}=10$ to 80 stations for infrastructure network, operating at $80 \%$ offered load. I also observed that mean packet delay under the infrastructure network, especially for $\mathrm{N}>10$ stations, has more delay. The main conclusion that could be drawn from Fig. 9 is that the network mean packet delay performance under the IEEE 802.11b WLAN deteriorates as the number of workstations on the network increases, especially at high offered load. Therefore, the IEEE 802.11b WLAN may not be a suitable standard for real-time applications (e.g. multimedia communication) because the mean packet delay is very large for $\mathrm{N} \geq 20$ stations at high traffic loads. The reason is that the frame delay in each station originates from the back off defer periods in a significant portion, as well as from the periods when the station participates in collisions.

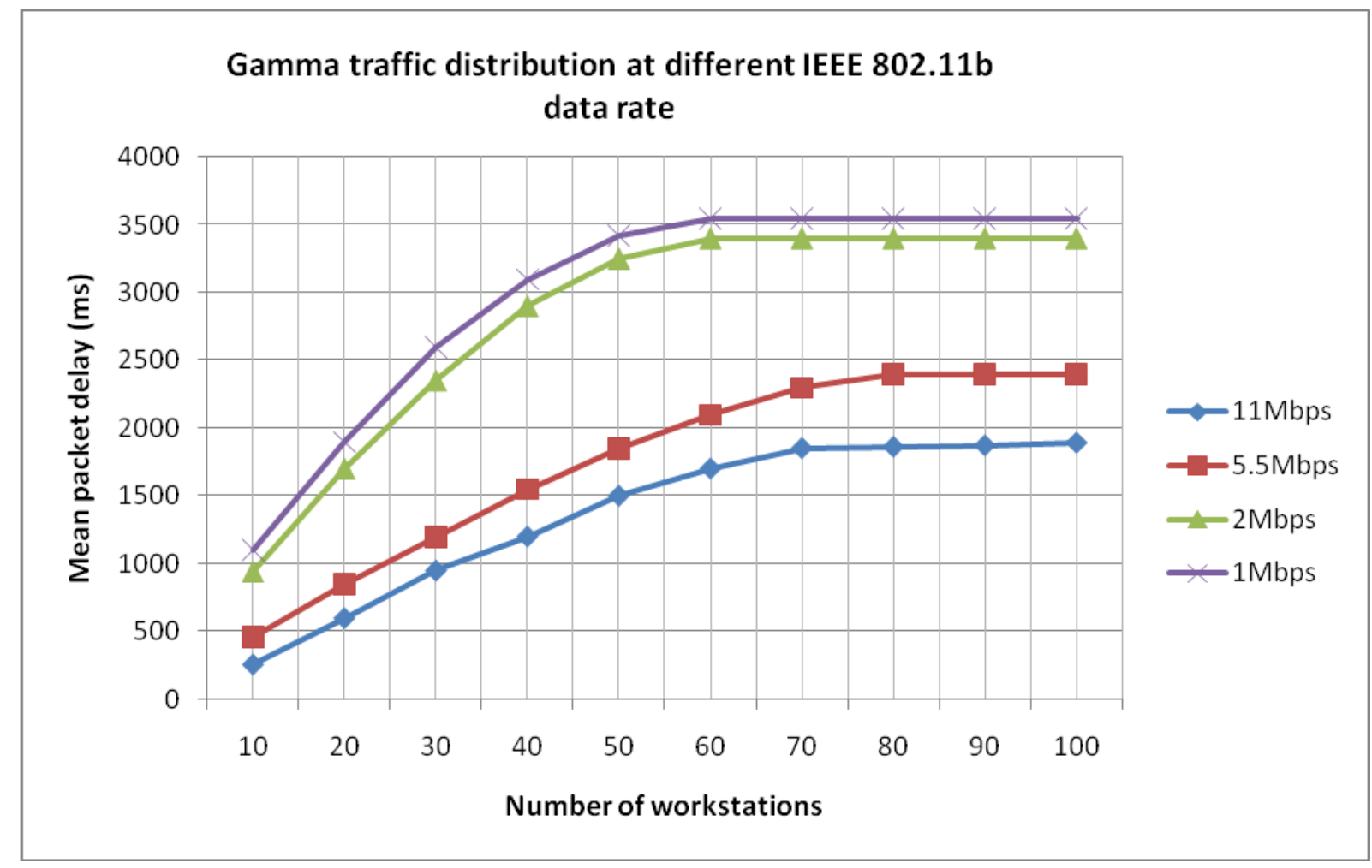

Figure 9: Graph of Mean Packet Delay against Number of Sources using Gamma traffic distribution 
Considering the Gamma traffic distribution, which has the best performance and slightly different behaviour from the three distributions, showed that the mean packet delay increased continously at medium traffic load from $10-70$ workstations at both 5.5 and $11 \mathrm{Mbps}$ and at 1 and 2 Mbps, mean delay increased from $10-60$ workstations whereas at high traffic load, the mean delay at different data rate behave alike from $80-100$ work stations at $11 \mathrm{Mbps}$, $80-100$ workstations at 5.5Mbps, and $70-100$ workstations at 1 and $2 \mathrm{Mbps}$.

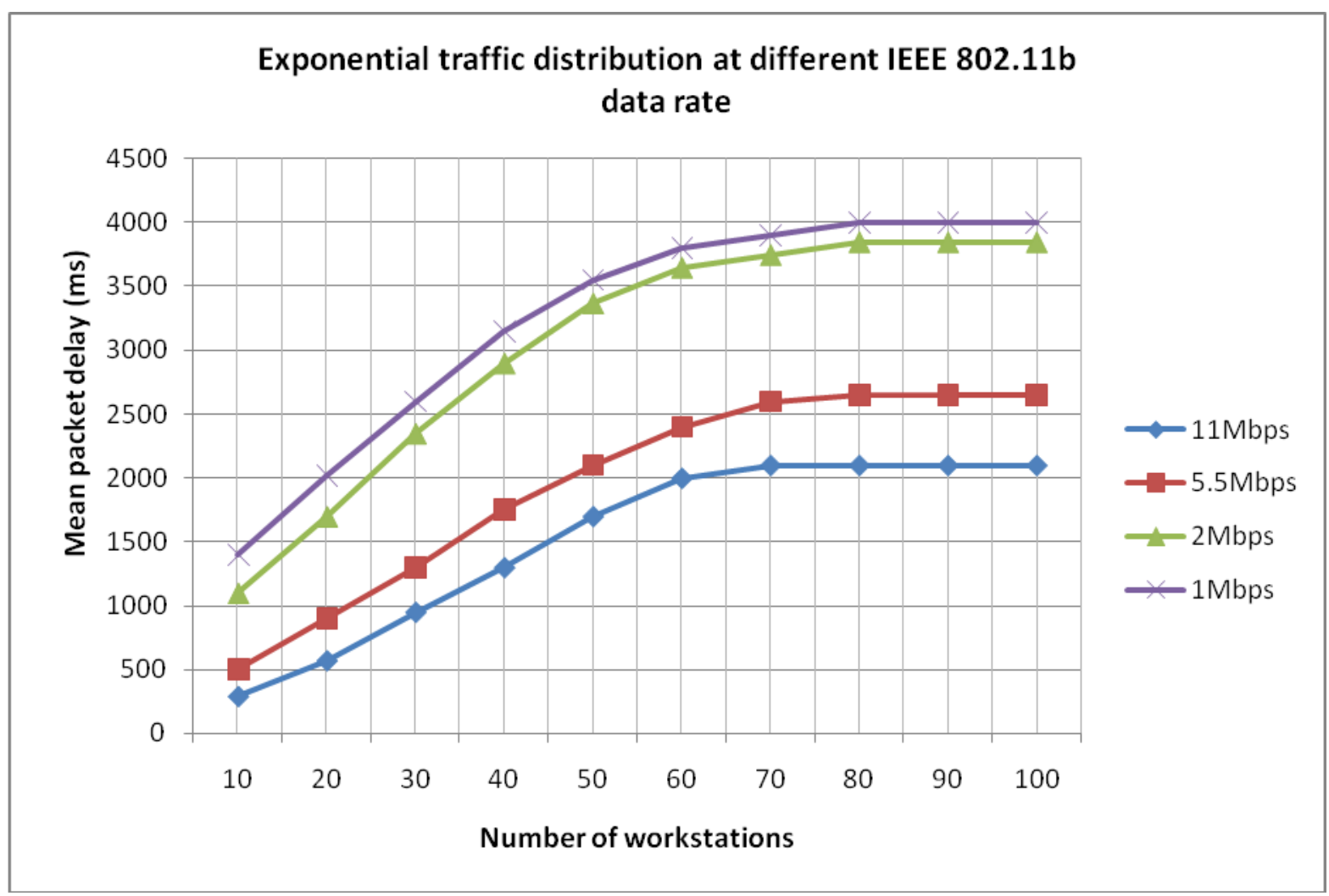

Figure 10: Graph of Mean Packet Delay against Number of Sources using Exponential traffic distribution 


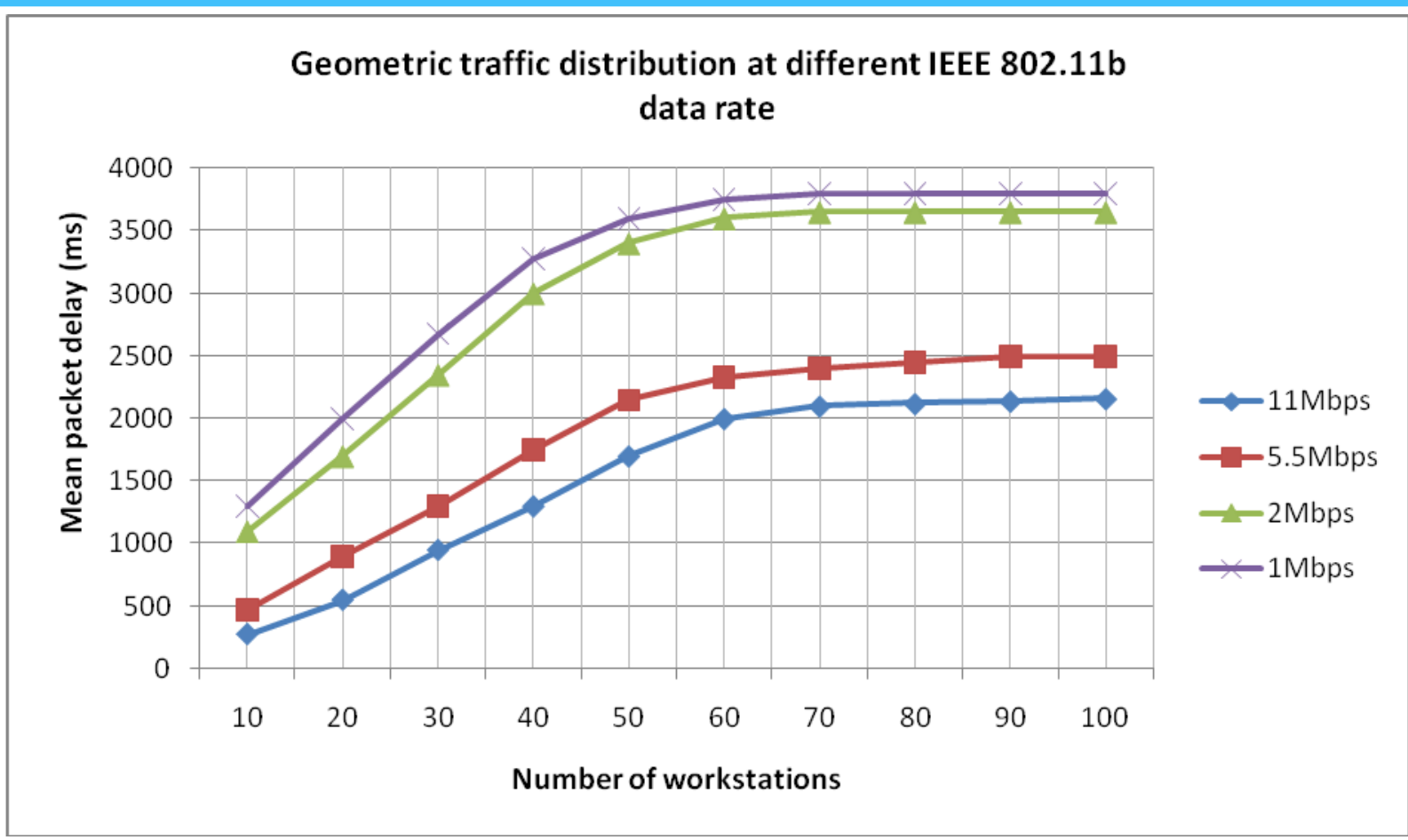

Figure 11: Graph of Mean Packet Delay against Number of Sources using Geometric traffic distribution

\subsection{Ways of Enhancing the QOS}

\subsubsection{Differentiation Serving System}

Differentiation serving bases on the thought that different requirements of different applications should be served distinctively in different classes. There are two modes of differentiation service In IEEE 802.11 : priority based service and fair scheduling service. The classification mechanism identifies and separates traffics into different flow. Therefore, each flow can be handled selectively. Priority based service always serve those flows with the highest priority in the remaining ones. Fair scheduling service fairly schedules the bandwidth on the basis of weight of each flow, so it can avoid that lower priority flows cannot get service all the time.

\subsubsection{Information Extracting System Based on PHY Layer}

If we can get the exact status report of transmission environment periodically from $\mathrm{PHY}$ layer, some parameters of MAC layer can be tuned to achieve maximum throughput and minimum delay, in another words, it needs a information extracting system based on PHY layer of dynamic wireless environment to optimize QOS guarantee.

\subsubsection{Tuning IEEE 802.11 Parameters}

Tuning parameters defined in IEEE 802.11 will make great effects on performance of WLAN. These parameters are listed out in the Table 1. "summary of common tuneable parameters." As in the table 1, RTS threshold is a parameter frequently being quoted in research. It is the conclusion in that RTS-CTS system is especially efficient when there are long MSDU packets needs to be transmitted. Some author suggests that it is better to set RTS threshold as 0 that is using RTS-CTS system all the time than work out the amount of active STAs to adjust RTS threshold dynamically. In addition, fragmentation threshold is also a parameter worthy of further study. To get more throughputs in bad wireless environment, it'd 
better adjust RTS threshold dynamically based on the channel quality feedback from lower layer. Dynamical tuning Long Retry Limit and Short Retry Limit also can be used to optimize WLAN's throughput and data rate.

Table 1: Summary of common tuneable parameters

\begin{tabular}{|c|c|c|c|}
\hline Parameters & Meaning and Unit & Effect when Decreased & Effect when increased \\
\hline RTS Threshoold & $\begin{array}{l}\text { Frames larger than the } \\
\text { threshold are preceded by } \\
\text { RTS/CTS exchange }\end{array}$ & $\begin{array}{l}\text { Greater effective throughput if } \\
\text { there are a large number of } \\
\text { hidden node situations }\end{array}$ & $\begin{array}{l}\text { Maximum theoretical throughput } \\
\text { is increased, but an improvement } \\
\text { will be realized only if there is no } \\
\text { interference. }\end{array}$ \\
\hline $\begin{array}{l}\text { Framentation } \\
\text { Threshold }\end{array}$ & $\begin{array}{l}\text { Frames larger than the } \\
\text { threshold are transmitted } \\
\text { using the fragmentation } \\
\text { procedure }\end{array}$ & $\begin{array}{l}\text { Intereference corrupts only } \\
\text { framgments, not whole frames, so } \\
\text { effective throughput }\end{array}$ & $\begin{array}{l}\text { Increases throughput in noise-free } \\
\text { areas by reducing framnetation } \\
\text { acknolwedgment overhead }\end{array}$ \\
\hline
\end{tabular}

\subsection{CONCLUSION}

The objective of this research is to carry out a performance analysis of IEEE $802.11 \mathrm{~b}$ medium access control distributed co-ordination function traffic loading under network saturation by investigating the effect of traffic distribution on the quality of service parameter on WLAN, in other to establish enhanced WLAN Performance. In order to evaluate the mean packet delay and network throughput performance, this research has presented a traffic loading for MAC DCF which supports WLAN's QOS Parameters. Using the proposed model, evaluation of the mean packet delay and network throughput performance of IEEE802.11b DCF for basic access mechnism under network saturation condition using MATLAB. Based on this model, a computer simulation model over a MATLAB Simulink (Simevent Environment) was developed, which is characterized by a MAC DCF protocol that enhances station's transmission probability and fair access to the channel, and more realistic, such as non ideal channel conditions. In other words, The basic access mechanism is a key feature of the IEEE 802.11b standard that uses virtual carrier sensing and random back off procedure to avoid the probability of two or more station from accessing the channel at the same time, thus, causing collision. To realize this feature, the WLAN MAC architecture design is organized, modeled, and implemented in the Simevent within the MATLAB Simulink framework, in an organized manner following the software engineering best practices.

In this research, Results show that the IEEE $802.11 \mathrm{~b}$ does not perform well in terms of high throughput, and low mean delay at high traffic load conditions. For example, if the number of workstations increases, both mean packet delay and throughput performance of the IEEE 802.11b protocol degrades significantly. Similarly, it was also shown that the mean packet delay of arrived packets decreases as the number of workstations decreases, but at saturation, it was shown that throughput decreases, mean packet delay increases. Clearly, the existing IEEE 802.11b WLANs cannot be used for high bandwidth real- time applications serving large number of users. Therefore, to achieve an enhanced network performance, it was observed that the IEEE 802.11b WLAN requires an improvement on its window back off algorithm and fairness. 


\section{REFERENCES}

Alberto Leon- Garcia, Indra Widjaja, 'Communication Networks'; Fundamental concepts and key Architectures. Page 422-427. Copyright 2000.

Dedric Carter, Andrew Garcia, David Pearah (MIT) Stuart Buck, Donna Dutcher(Harvard),"Spread Spectrum: Regulation in Light of Changing Technologies" 1998

Eun-Sun Jung and Nitin H. Vaidya, Energy Efficient MAC Protocol for Wireless LANs," in Proc. IEEE INFOCOM’ 02, New York, U.S.A., June 2002.

Frank.H.P.Fitzek, D.Angelini, G.Mazzini, and M.Zorzi, "Design and Performance of an Enhanced IEEE 802.11 MAC Protocol for multihop coverage Extension", Proceedings IEEE Wireless Communication, vol. 10, No. 6, Dec., 2003,pp. 30.

G. Bianchi and Ilenia Tinnirello, "Kalman Filter Estimation of the Number Of Competing Terminals in an IEEE 802.11 Network," in Proc. IEEE INFOCOM'03, San Francisco, U.S.A., April 2003.

G. Bianchi, "Performance Analysis of the IEEE 802.11 Distributed Coordination Function," IEEE Journal of Selected Areas in Communications (JSAC), vol. 18, no.3, March 2000

G. Held, "Data communications networking devices;operation, utilization and LAN and WAN internetworking,"fourth edition, pp 271-277, copyright 2001 ,John wiley and sons Ltd.

IEEE 802.11 WG. IS0/IEC 8802 - 11: 1999 (E). International standard for information technology - telecommunications and information exchange between systems - local and metropolitan area networks - specific requirements - part 11: wireless LAN medium access control (Mac) and physical layer (phy) specifications. 1999.

Jaehyuk Choi, Joon Yoo, Sunghyun Choi, Member, IEEE, and Chongkwon Kim,Member, IEEE "EBA: An Enhancement of IEEE 802.11 DCF via Distributed Reservation", IEEE transactions on mobile Computing vol. 1, no.3, March 2002

Omar Villavicencio-Calderon, Kejie Lu." Wireless Mesh Network: Performance Analysis and Enhancements" 2008.

P.Padhye J.,Wolman A, Lidong Z.Adya, A.Bahl. A multi-radio unification protocol for IEEE 802.11 Wireless network.In broadband networks,2004. BroadNets 2004.Proceedings. First International Conference on Pages 344-354. 2004

S. Herkmat,"communication network", Pragsoft Corporation, www.pragsoft.com, pp., 109-112, copyright 2005.

S. Mangold, S.Choi, P.May, O.Klein, G.Hiertz,and L.Stibor. IEEE802.11e Wireless LAN for quality of service (invited paper). Volume 1, Pg. 32-39 \{Florence, Italy\} Feb., 2002 
Sanjay Sharma, "Wireless Communication”, S.K. Kataria, 2007, pp 167-170.

Standard for WLAN Medium access control (MAC) and physical layer (PHY) Specification. Medium access control (MAC) Quality of service IEEE Std 802.11 edition revised 2007.

Sunghyun Choi, "PCF vs. DCF Limitations and Trends," IEEE 802.11-01/054, January 2001.

T. S. Rapparport, Wireless Communications, Principles and Practice, Prentice Hall, 1996.

V. Bharghavan, A. Demers, S. Shenker, and L. Zhang, "MACAW: A Media Access Protocol for Wireless LAN's,” in Proc. ACM SIGCOMM’94, Sept. 1994.

W.L.Pang, K.W.Chew, Florence choong and E.S.Teoch,"VHDL Modeling of the CSMA/CA", Intrnational journal of Communications, issue 2, volume 1, 2007, pp. 81.

Younggoo Kwon, Yuguang Fang, and Haniph Latchman, "A Novel MAC Protocol with Fast Collision Resolution for Wireless LANs," in Proc. IEEE INFOCOM'03, San Francisco, U.S.A., April 2003. 